\section{Saberes médicos y reflexiones morales durante el período rosista: Buenos Aires, 1835-1847}

\section{Medical knowledge and moral reflections during the Rosas era: Buenos Aires, 1835-1847}

Mariano Di Pasquale ${ }^{i}$

' Investigador asistente y profesor adjunto, Instituto de Estudios Históricos/Universidad Nacional de Tres de Febrero-Consejo Nacional de Investigaciones Científicas y Técnicas (Conicet). Buenos Aires - Argentina

orcid.org/0000-0002-6198-9954

mariano.dipasquale@gmail.com
DI PASQUALE, Mariano. Saberes médicos y reflexiones morales durante el período rosista: Buenos Aires, 1835-1847. História, Ciências, Saúde - Manguinhos, Rio de Janeiro, v.26, n.3, jul.-set. 2019, p.733752.

\section{Resumen}

Este artículo analiza cómo el discurso médico incorpora una serie de reflexiones sobre las conductas morales en Buenos Aires en la primera parte del siglo XIX. A través del estudio de tres textos, cuyos autores son los médicos Diego Alcorta, Guillermo Rawson y Francisco Javier Muñiz se identifican una serie de registros argumentales que resaltan el funcionamiento de los órganos, la cuestión de la herencia y la gravitación del clima en función de reflexionar sobre la moralidad de los individuos y las poblaciones. Este fenómeno de transferencia de saberes se debe a la presencia de la tradición médica francesa sumándose a factores locales derivados del intenso proceso de politización de la sociedad bajo el segundo gobierno de Juan Manuel de Rosas.

Palabras claves: saberes médicos; moral; política; Buenos Aires; siglo XIX.

\section{Abstract}

This article analyzes how medical discourse incorporated a series of reflections on moral behaviors in Buenos Aires in the early nineteenth century. Based on the study of three texts authored by the physicians Diego Alcorta, Guillermo Rawson and Francisco Javier Muñiz, it identifies a series of discursive registers that stress the role of organ functions, the question of heredity and the influence of climate in reflections on the morality of individuals and populations.

This phenomenon of knowledge transfer is due to the presence of the French medical tradition, in addition to local factors stemming from the intense process of politicization of society under the second administration of Juan Manuel de Rosas.

Keywords: medical knowledge; moral; politics; Buenos Aires; nineteenth century. 
$\mathrm{E}^{\mathrm{n}}$ n el Río de la Plata, durante la primera mitad del siglo XIX, se dieron una serie de reflexiones por parte de los médicos "diplomados" en donde intercalaron cuestiones referidas a los comportamientos sociales y morales del hombre. Pero esto no es extraño en una época en donde la medicina estaba en tensión entre mantenerse como un saber humanístico o constituirse como un saber empírico-experimental. La etapa que analizamos corresponde a un momento en el que la medicina, aún en Europa, se reivindicaba dentro de un arte de curar y no como un saber experimental, como sí lo hizo a partir de Claude Bernard (Ramsey, 1988; Haber, 1991; Vigarello, 1993; Bonner, 1995; Grmek, 1997; Gaudillière, 2006; Bynum, 2006; Lindemann, 2010).

En tal sentido, varios médicos porteños, siguiendo de cerca las referencias y los aportes de Pierre Jean Georges Cabanis, Xavier Bichat, François Magendie y Philippe Pinel - sus partes en Francia -, concibieron que en materia médica era necesario estudiar aquellos aspectos físicos y orgánicos del hombre junto con los morales. Pero además de este fenómeno de recepción de la medicina francesa, existieron cuestiones locales, las derivadas del intenso proceso de politización que se dio durante la etapa rosista, que acentuaron el "uso" de estos saberes médicos provocando que fueran tomados como fuentes válidas para ensayar explicaciones de índole moral.

El propósito del presente artículo es indagar con más profundidad sobre cómo la medicina ofreció una serie de reflexiones sobre las conductas morales a partir del estudio de tres textos producidos por médicos locales. El análisis está centrado en las Lecciones de filosofía de Diego Alcorta (1835); en la Disertación para obtener el grado de doctor en medicina de Guillermo Rawson (1845) y, finalmente, en los "Apuntes topográficos del centro de la provincia de Buenos Aires" de Francisco Javier Muñiz (1847) (ver Alcorta, 2001; Rawson, 1845; Muñiz, 1944). Si bien las temáticas que se encuentran en estos textos son variadas, los hemos escogido porque comparten la propuesta de presentar a la medicina como un insumo válido y viable para comprender mejor aquellos aspectos morales de los hombres.

Este trabajo se inserta dentro de una perspectiva teórica que busca abordar "lo local" como una fructífera categoría analítica, al proponer las ventajas de las "historias situadas" de la ciencia y hacer referencia a ella como una actividad práctica (Van Damme, 2010, p.242-254; Pestre, 2005, p.487-522; Pickering, 1992). Desde esta aproximación, se observa que en estos textos convergen una serie de saberes provenientes de la tradición higiénica, el sensualismo, el vitalismo (École de Montpellier) y la fisiología francesa, pero fueron adaptados y situados a partir de la realidad local.

La historiografía local ha remitido para las últimas décadas del siglo XIX y comienzos del siglo XX lo que suele calificar como el "momento higienista" y/o el "momento alienista/psiquiátrico", etapa que coincide con la recepción de un positivismo materialista y evolucionista, con la existencia de un proceso de profesionalización de la actividad médica y, a su vez, con la incorporación de los médicos en tanto funcionarios calificados en cargos públicos del Estado-nacional en pleno proceso de consolidación (Armus, 2007; Di Liscia, 2002; Di Liscia, Salto, 2004; González Leandri, 1999; Zimmermann, 1992). Siguiendo de cerca estos avances, nos parece importante completar el panorama teniendo presente la situación del período previo, identificando algunos casos representativos en pos de resaltar que existieron importantes "huellas" allí. Las interrelaciones entre el mundo 
del conocimiento y el mundo del poder político resultarían más tempranas en el caso de aquellos que se vieron ligados a la medicina, no solo porque participaron en la vida pública, sino porque - y esto será en lo que nos concentraremos aquí - brindaron un conjunto de intervenciones escritas en donde los saberes médicos constituyeron fundamentos para comprender las cuestiones morales de los individuos y los grupos humanos.

\section{La vida académica y política de los médicos en la época de Rosas}

Es conveniente comenzar dando algunas características generales del contexto político e indicar la situación de la vida universitaria bajo las cuales transcurrieron las vidas de los tres galenos escogidos en este trabajo. Las trayectorias personales de Alcorta, de Muñiz y de Rawson estuvieron cruzadas por el momento en el que Juan Manuel de Rosas llegó a la gobernación de la provincia de Buenos Aires, en 1829 hasta 1832, y por su segunda gobernación, entre 1835 y 1852.

De las características principales del sistema político rosista se podría destacar la continuidad del Ejecutivo y la centralización del poder. Como indica Marcela Ternavasio (1999, p.119-141; 2002, p.204-205), el gobierno rosista no desarticuló el andamiaje republicano y representativo introducido por las reformas rivadavianas (1821-1824), sino que fue reordenado bajo la modalidad unaminista y plebiscitaria. Asimismo, durante la primera y segunda gobernación de Rosas se produjo una participación activa de los sectores populares y una alta politización del conjunto social. La beligerancia permanente contra todos los intentos de oposición, sumado a las guerras entabladas con potencias extranjeras, generaron una situación de constante inestabilidad política (Myers, 1995, p.20).

En este contexto particular se insertó la vida intelectual y académica. El proceso de politización, sumado a las guerras internas y externas a lo largo del período, tuvo un impacto significativo en la Universidad de Buenos Aires, en cuyos recintos se experimentaron una serie de divisiones y conflictos (Buchbinder, 2005, p.48-49). Los médicos que se formaron en estas aulas conocieron de cerca estas circunstancias de la vida pública y, entre otras formas, participaron de ella, reflexionando y dejando por escrito apreciaciones referidas a la moral y a la vida política y social. En tal sentido, los contenidos que subyacen en los textos que analizaremos se conectan con este proceso de politización de la sociedad porteña.

Tres figuras ejercieron el rectorado de la Universidad de Buenos Aires entre $1830 \mathrm{y}$ 1852: los doctores Santiago Figueredo, Paulino Gari y Miguel García. El primero se doctoró en derecho civil y canónico en la Universidad de Córdoba y rigió la Universidad desde agosto de 1830. Gari se doctoró en Córdoba y en Charcas, y fue designado rector por decreto del 13 de diciembre de 1832, cargo que ya venía desempeñando interinamente por fallecimiento de Figueredo. El doctor García, que estudió derecho y teología en la Universidad de Trejo y en Charcas, dirigió la alta casa de estudios porteña desde diciembre de 1849 hasta junio de 1852 (Gutiérrez, 1998, p.441).

Al finalizar el año 1832, Rosas dejó el poder sin haber tomado ninguna medida de importancia con respecto a la Universidad durante todo el curso de su primer gobierno. Ésta conservó su estructura anterior, tal como venía funcionando hasta entonces. Al año siguiente, por fallecimiento del doctor Figueredo, el doctor Gari es nombrado rector de la 
Universidad, cargo que desempeñó durante la mayor parte de la administración de Rosas. En ese año se procede a la reorganización. En tanto se procedió a esta reforma, Rosas se hallaba en plena campaña del desierto. El 17 de diciembre de 1833 el gobernador Viamonte aprobó la reforma universitaria proyectada por una comisión que integraron los doctores José Valentín Gómez, Diego Estanislao Zabaleta y Vicente López y Planes. Según este nuevo régimen, el gobierno de la Universidad pasaba a cargo de un Consejo Directivo que fue establecido por decreto del 15 de mayo de 1834 y lo integraban los siguientes catedráticos: de derecho canónico, doctor José León Banegas; de derecho civil, doctor Rafael Casagemas; de nosografía médica, doctor Cosme Francisco Argerich, y de ideología, doctor Diego Alcorta (Halperin Donghi, 1962, p.56).

De modo que al acceder Rosas por segunda vez al poder en abril de 1835, la Universidad de Buenos Aires contaba solamente con un año de experiencia en su nueva organización. Al poco tiempo, por decreto del 11 de mayo de ese año, el Consejo fue suprimido a instancias del rector Gari (Gobierno..., 1836b). Posteriormente, por decreto del 14 de diciembre, se fijó definitivamente la organización estructural de la Universidad, cuyo personal administrativo debía ser el siguiente: un rector, un secretario, un prosecretario y un celador general; el docente, se reducía en algunas cátedras que fueron suprimidas; y el de servicio quedaba constituido por un portero y un ordenanza (Gobierno..., 1836d).

A partir de los nombramientos que se realizaron en 1834 y 1835 se produjeron cambios en el personal docente, lo que generó ciertas tensiones al interior del cuerpo médico. Al respecto, Diego Alcorta (27 dic. 1834) escribe a su amigo Daniel Torres:

Los médicos, como ya calcularás, han formado dos facciones: una compuesta de Fernández, Montes de Oca, Manuel García, los estudiantes todos y varios particulares que los sostienen y son particularmente Pablo Salvadores, Arana, Baldonuero, y creo que los Anchorenas y Wright. La otra la forman Rivera, Montufar, Fuentes Arguivel y particulares Irigoyen, Mansilla, la Reina Madre y la princesa Mercedes. Argerich pertenece a esta última; pero no escribe.

Seguramente, el objeto inicial de esta disputa guardó relación con las salidas y llegadas de catedráticos designados por el gobierno. Además, debe agregarse que, durante junio de 1835, se estableció el decreto de juramento de fidelidad a la causa "Nacional de la Federación" (Gobierno..., 1836c). Más tarde, se dio a conocer el decreto del 27 de enero de 1836, cuyo artículo 1, indica que:

A nadie se podrá conferir en la Universidad el grado de Doctor en ninguna facultad, ni expedírsele título de Abogado o Médico, sin que previamente haya acreditado ante el Gobierno, y obtenido sobre ello, la correspondiente declaratoria de haber sido sumiso y obediente a sus Superiores en la Universidad durante el curso de sus estudios, y de haber sido y ser notoriamente adicto a la causa nacional de la Federación (Gobierno..., 1836e).

Este tipo de disposiciones tenían sus antecedentes. En febrero de 1832, por decreto de Rosas, se dispuso el uso obligatorio de la cinta punzó como símbolo de la Federación. Era pues una forma de hacer visible e identificar la pertenencia al régimen. Al principio la medida alcanzó solo a los funcionarios públicos, pero luego se extendió a toda la sociedad. 
Quienes no acataban estas medidas quedaban al margen de la comunidad, y, por ende, eran considerados enemigos de la patria (González Bernaldo de Quirós, 2008, p.233-234). Estos decretos buscaron generar manifestaciones obligadas de pertenencia al gobierno. De tal modo, podemos inferir que durante el período rosista existió una fuerte presencia y control en la regulación de la vida universitaria y académica. Los tipos de vínculos sociales establecidos y la misma actividad profesional se encontraron atravesados por las lógicas de poder y los acomodamientos políticos.

Por otro lado, se sumó la necesidad de ajustar el presupuesto universitario para cubrir el déficit que afectaba a la provincia. En los tres primeros años del segundo gobierno de Rosas, la Universidad desarrolló sus actividades sin inconvenientes, pero al llegar el año 1838 se determinó la adopción de serias medidas que alteraron su funcionamiento. A fines de marzo de 1838, el contralmirante Leblanc, comandante de la escuadra francesa, declaró el bloqueo del puerto de Buenos Aires y de todo el litoral fluvial, lo que vino a sumarse a la guerra que la Confederación Argentina mantenía, desde diez días antes, con la Confederación Peruano-Boliviana. A partir de entonces, la Universidad quedó privada de suficiente apoyo económico y los alumnos debieron abonar una cuota mensual. Para sobrellevar esta situación algunos profesores acordaron dictar gratuitamente sus clases (Gutiérrez, 1998, p.449).

Estos problemas en conjunto tuvieron como consecuencia cierta desorganización de los estudios universitarios y la polarización de las distintas posiciones que afectó la vida personal y profesional de un número importante de médicos. En 1836, Cosme Francisco Argerich, Juan Antonio Fernández y Juan José Montes de Oca, en distintas circunstancias, se opusieron a Rosas, se alejaron de la enseñanza y debieron emigrar a Montevideo. En cambio, otros médicos siguieron los vaivenes de la política rosista como el doctor José María Gómez de Fonseca que siendo miembro del Tribunal de Medicina desde 1833 fue destituido por Rosas en 1835 por no considerarlo hombre de confianza de su gobierno (Fonseca se había opuesto a las facultades extraordinarias), pero luego, el gobernador reconsideró el caso y, en 1836, lo designó profesor de clínica quirúrgica, cátedra que desempeñó hasta su muerte, el 31 de noviembre de 1843 (Yrigoyen, 24 abr. 1835). Otro caso interesante fue el del médico y poeta Claudio Manerto Cuenca que, aunque contrario a Rosas, terminó siendo su médico personal. La trayectoria de Cuenca representaría una forma de estrategia que tuvieron algunos médicos y hombres ligados al conocimiento que optaron por no abandonar Buenos Aires haciendo uso de la simulación.

A pesar de los controles del gobierno y de los recortes presupuestarios, la vida universitaria de los médicos como objeto de atención parece resultar un fenómeno más complejo y variado de lo que la historiografía local ha sugerido. Esta diversidad del universo médico puede pues constatarse tomando como muestra representativa a Diego Alcorta, Guillermo Rawson y Francisco Javier Muñiz ya que éstos tuvieron distintos momentos de encuentros y desencuentros respecto del gobierno de Rosas, representando distintas maneras de inserción profesional y relacionamiento político.

Alcorta tuvo un paso fugaz por la política. Fue electo como diputado para la Sala de Representantes de Buenos Aires por el partido de San Isidro en 1832. Desde el cargo, se opuso a la reelección de Rosas en 1834. Luego, decidió abandonar los cargos públicos centrándose 
en la enseñanza y en el ejercicio de la medicina. Es muy probable que este alejamiento haya sido la razón para sostenerse en la Universidad, en la cátedra de ideología, hasta 1842.

En cambio, Guillermo Rawson se enfrentó más abiertamente a Rosas como lo demuestra su acción político-militar en San Juan para lograr remover a Nazario Benavidez, gobernador federal afín al gobernador porteño. El caso de Rawson es representativo de cómo un médico logra trazar una carrera cada vez más cercana a la toma de decisiones políticas: fue legislador por su provincia en 1844, luego fue nombrado diputado de la confederación en 1856; pasó por los cargos de senador provincial y nacional entre 1861 y 1862 para finalmente terminar siendo, ese año, ministro del interior de Bartolomé Mitre.

Por último, Francisco Muñiz tuvo cierta afinidad respecto del proyecto político de Rosas. Durante el segundo gobierno, fue nombrado médico de policía para vigilar la sanidad de la población y de sus animales y controlar el ejercicio de la medicina y sancionar el curanderismo. Una muestra de esta cordial relación es el hecho que hacia 1841 Muñiz decida regalarle su colección paleontológica. Fue también el médico personal de Rosas, a quien trataba por una afección prostática, y fue nombrado conjuez del Tribunal de Medicina. En la batalla de Caseros, figuró como asistente del cirujano jefe del ejército rosista, el doctor Claudio Mamerto Cuenca, y se encargó del envío del material médico necesario para la asistencia de los heridos. Tras esta cantidad de experiencias profesionales, entendemos mejor el hecho de que, en 1853, Muñiz haya sido electo diputado del estado de Buenos Aires, por la sección de la campaña que comprendía Luján; y, al año siguiente, senador provincial.

Las trayectorias de Alcorta, de Rawson y de Muñiz nos muestran distintas modalidades de acomodamiento que pudieron haber tenido los médicos frente al poder político de ese entonces. Más allá de las posturas y alineamientos, sus vidas se encontraron cruzadas por el mismo espacio politizado y faccioso. Y fue en este horizonte donde estos galenos pusieron en disponibilidad una serie de saberes médicos-fisiológicos en pos de reflexionar sobre los problemas de su tiempo. Con ello, entendieron que el mundo de lo moral podía (y debía) ser plausible de explicaciones médicas desprendiéndose que éste ya no era un asunto exclusivo de la filosofía o la teología.

\section{Diego Alcorta, de la sensibilidad a la imitación}

Entre las décadas de 1820 y 1830, se identifica la presencia de un conjunto de nuevos saberes que arribaron a la vida universitaria y, con ella, también ingresaron al Departamento de Ciencias Médicas. Por un lado, esta renovación resultó en la inauguración de la cátedra de ideología, en 1822, obligatoria para todos los estudiantes, y cuyo objetivo consistió en difundir las premisas de Destutt de Tracy y Jean Pierre Cabanis. Por el otro lado, en las materias que se dictaban en la carrera de medicina, se introdujeron conocimientos provenientes de dos vertientes médicas francesas: el vitalismo (o Escuela de Montpellier, en especial, Xavier Bichat) y la fisiología experimental (Escuela de París, en particular, François Magendie y Philippe Pinel).

La preocupación de la idéologie por destacar una preponderancia de los sentidos y la sensibilidad en las capacidades humanas se articuló fuertemente con la tesis del vitalismo, en particular con aquella que hacía referencia a la irreductibilidad de los procesos vitales, al 
estudio de los mecanismos físicos o químicos y que no podían ser objeto de experimentación, lo que los distinguía de la escuela de París que proponía François Magendie. De estos postulados surgió, tanto en Francia como en el Río de la Plata, la interacción entre lo físico y lo moral y el encuentro de la ideología con el vitalismo. ${ }^{1}$

Esta conexión generó un acercamiento entre la propuesta de Cabanis y la de Bichat, a la que se sumó el compartir la importancia de la observación clínica y la disección como métodos de aprendizaje y ejercicio profesional. Pero, como veremos, no por ello se desestimó enseñar e incorporar las ideas de Pinel y las de Magendie que más bien pusieron el acento en un método médico asociado a la clasificación de las enfermedades (nosografía filosófica) y a la posibilidad de experimentar con sustancias químicas, respectivamente.

Las modalidades de acceso y circulación de estos conocimientos en Buenos Aires fueron variadas. Una vía de difusión de la ideología se produjo de forma indirecta a través de las enseñanzas que impartió Crisóstomo Lafinur en el Colegio de la Unión del Sud, en 1819, y luego con la creación de la cátedra ideología en la Universidad, a cargo de Juan Manual Fernández de Agüero entre 1822 a 1827. Lafinur publicó el Curso filosófico en 1819 mientras que Fernández de Agüero los Principios de ideología en 1824 (ver Lafinur, 1939; Agüero, 1940). Financiadas por el gobierno, estas obras se usaron como manuales para el dictado de clase. En ambas, aparecen diversas citas y explicaciones retomando las ideas de Cabanis, Destutt de Tracy, Condillac (Di Pasquale, 2011, p.63-86).

También se publicaron "extractos" de los libros originales traducidos por editores españoles, que, junto con comentarios, aparecieron en diversos periódicos porteños. Al respecto, La Abeja Argentina de 15 de abril de 1822 señala que "Las ideas de Magendie, de Bichat, de Richerand, de Alibert, de Pinel, de Tenard, de Orfila etc. hacen la base de nuestros cursos y de nuestras lecciones diarias" (La Abeja..., 1960). Este tipo de recepción hace pensar que los médicos franceses fueron conocidos especialmente a través de relecturas y de su repercusión en la prensa escrita, más que por el acceso directo a sus obras.

Sin embargo, se sabe que algunos libros de medicina se vendían en las librerías de Buenos Aires (Parada, 1998). Asimismo, algunos médicos locales tuvieron acceso directo a las obras de sus pares franceses ya que en sus bibliotecas particulares figuran ejemplares con datos que permiten inferir que tenían originales o bien traducciones del español como sucede en el caso de Cosme Mariano Argerich (1758-1820). En la lista del testamento de Argerich se registra, entre otros libros, el de Xavier Bichat, Investigaciones fisiológicas sobre la vida y la muerte, dos tomos; y el de Philippe Pinel, Medicina clínica (Argerich, 1820). ${ }^{2}$

En sus notas de viaje, el francés Arsène Isabelle dejó constancia de aquellos autores que se enseñaban en el área de medicina en la Universidad de Buenos Aires en la década de 1830. Los nombres que señala coinciden con los médicos que estamos indicando, a saber: "anatomía: Maigrier; fisiología: Magendie; materia médica: Alibert; nosografía médica: Pinel; patología general: Caillot; higiene: Rostand" (Isabelle, 2001, p.300). Entre viejas y nuevas enseñanzas, entre lecturas fragmentarias y completas - a veces, traducidas; en otras ocasiones, originales -, entre las novedades e información que ofrecía la prensa y los contactos interpersonales, se formaron pues Diego Alcorta, Guillermo Rawson y Francisco Muñiz.

Diego Alcorta cursó sus estudios en el Colegio de la Unión del Sud donde asistió a las lecciones de filosofía a cargo del profesor Crisóstomo Lafinur, quien le habría despertado 
gran interés por conocer mejor las obras de Destutt de Tracy y las de Cabanis (Gutiérrez, 1998, p.104). En 1824, durante su época de estudiante, fundó, junto a un grupo de compañeros, la Sociedad Elemental de Medicina, en donde cada uno de los socios obtenía un número de orden equivalente a su sitial académico. Se realizaban sesiones semanales en la que cada uno de sus miembros presentaba diferentes temas. De esta forma, Alcorta (1821) expuso sobre afecciones patológicas combinadas de los órganos y sobre las variedades del pulso arterial.

Posteriormente fue nombrado practicante mayor rentado del Hospital General de Hombres. Parece que tuvo una precaria situación económica, según nos cuenta el propio Alcorta. En una carta enviada al rector de la Universidad, Alcorta le expresa que "habiendo dado todas las funciones preliminares al recibimiento del grado de doctor en las facultades de cirugía y medicina, se halla en la imposibilidad de hacerlo por no tener el dinero necesario para el depósito" y le pide pues que "se sirva concederle el grado de doctor gratis" (Gutiérrez, 1998, p.610). Tras otorgarle esta solicitud, en 1827, Alcorta finalmente presentó su tesis, Disertación sobre la manía aguda, recibiendo el título de doctor en medicina.

En la tesis de Alcorta es posible advertir la presencia de la idéologie, el vitalismo y la fisiología francesa como hemos demostrado en un artículo anterior (Di Pasquale, 2014a). Cabanis sostiene que los órganos imprimen las sensaciones de los objetos y, por lo tanto, son los que producen las ideas y los sentimientos del hombre (Cabanis, 2005, v.1, p.203214). ${ }^{3}$ El esquema de las sensaciones que Alcorta $(1827$, p.8) incorpora es una derivación del expuesto por el médico francés, cuando indica que: "Las distintas épocas de la vida vienen acompañadas del desarrollo particular de algunas de las facultades intelectuales, y de ciertos sentimientos interiores nacidos del estado actual de los órganos de la economía".

Por otro lado, Alcorta recupera la clasificación de las enfermedades mentales elaborada por Philippe Pinel en su Traité médico-philosophique sur l'aliénation mentale. ${ }^{4}$ En esta obra, el médico francés distinguía la melancolía simple (delirio parcial), la manía (delirio generalizado con agitación), la demencia (debilitamiento intelectual generalizado) y la idiocia (perturbación total de las funciones intelectuales). Consideraba a las enfermedades mentales como un desarreglo de las facultades cerebrales causado por cierto número de causas: estas podían ser físicas (directamente cerebrales o simpáticas); heredadas; y morales, a las que le atribuye más de la mitad de los casos, que se pueden separar en las que derivan de las pasiones intensas y las que provienen de los excesos de todo tipo (Pinel, 1801, p.135137). Al respecto, Alcorta (1827, p.6-7) señala que:

Es necesario aislar los objetos para poder conocerlos bien. He ahí la necesidad de una clasificación en las enfermedades mentales; y no pudiendo hacerla por las alteraciones orgánicas que las ocasionan, por no ser bien conocidas, es preciso hacerla por los síntomas que las caracterizan; a mi juicio, la de Pinel merece la preferencia. El distingue la enajenación mental en cuatro especies distintas: manía, melancolía, demencia e idiotismo; cada una de estas especies es susceptible de infinitas variedades.

Acepta la terapéutica del "tratamiento moral" de Pinel que consistía en aplicar al enfermo una serie de ingenuos ardides como trabajos agrícolas o escuchar música para demostrar lo errado de sus juicios, a las que se agregaban otro conjunto de medidas como sangrías para 
la retención de sangre menstrual o hemorroidal, purgantes suaves o baños templados. Pero también la cuestiona por no incorporar los saberes de la anatomía patológica, es decir, no realizar una autopsia, "por creer imposible el establecer una relación entre las apariencias físicas manifestadas después de la muerte y las lesiones de las funciones intelectuales que se han observado durante la vida" (Alcorta, 1827, p.5).

A partir del 1828, el galeno fue designado profesor de la cátedra de ideología en la Universidad de Buenos Aires, en reemplazo del clérigo Luis José de la Peña - quien a su vez había sucedido a Fernández de Agüero -, manteniendo el cargo hasta 1842, año de su muerte. En este marco, confeccionó una guía o manual para el dictado de las clases denominada Lecciones de filosofía, obra editada posteriormente en 1835.

El cruce entre los saberes médicos y las cuestiones morales se aprecia en las Lecciones cuando Alcorta (2001, p.73) señala que "los mejores libros de moral son los de medicina". El médico porteño parte de que el hombre como individuo está dotado de acción y el primer objeto de todas las acciones es la tendencia hacia la felicidad que es inherente a la naturaleza humana (p.63). Cabe señalar que los sensualistas franceses, al igual que los utilitaristas británicos, asientan la moral en la felicidad o el interés. De esta consideración inicial se sigue que el hombre debe aumentar, emplear y conservar sus facultades. Primero, el hombre debe desarrollar las fuerzas físicas (en especial su salud, sin ella no hay felicidad); y luego aumentar las capacidades intelectuales (p.70-73).

Ahora bien, ¿qué sucede con el hombre y su búsqueda de felicidad con los deberes que implica vivir en sociedad? Alcorta explica que existe una necesidad de agruparse, el hombre es social por naturaleza. Hay un contrato tácito entre los pueblos y cada individuo, de la felicidad recíproca de cada contratante se deriva la felicidad común. Existen tres elementos que reglan la conducta social, estos son: la autoridad política, las leyes y las opiniones de los demás (Alcorta, 2001, p.74).

Siguiendo a Condillac (1877) y a Cabanis (2005, v.2, p.469-504), Alcorta (2001, p.82) introduce la noción de imitación en cuanto a que ella era importante en la formación y la educación de los hombres como se sigue a continuación:

El ejemplo determina el juego de nuestros órganos, dirige las acciones morales, y decide hasta de las opiniones. Así el niño habla imitando a su nodriza; la virtud se corrompe con la frecuentación del vicio, y el hombre se hace razonable o crédulo según las ideas que circulan alrededor de él.

De esta manera, el médico porteño distingue una "imitación peligrosa o viciosa", la de apegarnos a las ideas ajenas con más tenacidad que las formadas por uno mismo y una "imitación conveniente o útil", surgida del entendimiento que elije aquellas ideas atractivas y/o del ejemplo propio. El hombre debe apreciar lo que otros le dicen o enseñan, discutir con ellos, y rechazar sus errores, pero apropiar sus descubrimientos. De tal manera, Alcorta pone en relación la sensibilidad y el funcionamiento de los órganos con aquella premisa de la imitación "buena" o "mala" que opera en el desenvolvimiento de los comportamientos morales. La moral resulta pues así concebida como fisiología aplicada. 


\section{Guillermo Rawson, la cuestión de la herencia y la predisposición}

Otro médico significativo que incursionó en el análisis de los aspectos morales fue Guillermo Rawson que nació el 24 de junio de 1821 en la ciudad de San Juan. Allí transcurrió su infancia y adolescencia para luego trasladarse a Buenos Aires para continuar sus estudios en el Real Colegio de San Carlos (Cutolo, 1958-1985, p.71). Con tan solo 19 años descubrió los principios básicos del telégrafo, cinco años antes de que Samuel Morse transmitiera su primer mensaje; esto ocurrió en una clase de electricidad, en la que le planteó al Padre Gomila, su profesor, que mediante la transmisión de descargas eléctricas que tuvieran un significado convencional se podrían transmitir palabras a grandes distancias, y que "yo podría conversar con mi padre, que está en San Juan" (Rawson, 1927, p.19). Tras finalizar sus estudios, Rawson ingresó a la facultad de medicina de la Universidad de Buenos Aires. Tuvo como profesores a Claudio Mamerto Cuenca, a Teodoro Álvarez, a Martín García y a Juan José Fontana, entre otros, y compartió su aprendizaje con Pedro Ventura Bosch.

Las aptitudes demostradas por Rawson impulsaron a sus profesores, quienes mediante una carta le pidieron al rector Gari que se le entregara el grado de doctor en medicina sin rendir la tesis, amparándose en el artículo 13 del decreto del 21 de junio de 1827, que permitía que la universidad le otorgase este grado a todo aquel que fuese "ilustre y eminente en alguna facultad" (Gobierno, 1836a).

El rector no aceptó, ya que consideraba que lo que se le pedía se encontraba fuera de sus atribuciones; sin embargo, le encomendó al profesor Cuenca que, tras la lectura de la disertación de Rawson, le dirigiese la palabra en nombre de la facultad y le entregase el título de médico (Cuenca, 1845). De esta forma, Rawson se recibió en 1844, con una tesis que tenía como tema la transmisión de las facultades fisiológicas y patológicas en el hombre por vía de la herencia, la cual fue publicada en 1845. El tema elegido, la cuestión de la herencia, era una de las incógnitas más importantes de la época.

En la tesis de Guillermo Rawson (1845, p.25) se observa el eco de los saberes fisiológicos y del vitalismo cuando señala que las facultades inteligentes y morales se hacen comprensibles en el marco de lo que denomina "comunicación vital fisiológica". Rawson parte ubicando al cerebro como el órgano material del pensamiento en donde las distintas facultades del hombre están representadas cada una por una porción de la masa encefálica. Esta afirmación concuerda también con la de otros médicos locales, como Diego Alcorta. Evidentemente, el vitalismo de la escuela de Montpellier, cuestionado y pasado de moda ya para esta época en Francia, seguía siendo un importante referente en el plano de la medicina local. Pero resulta que Rawson más adelante en su exposición despliega una serie de fundamentos y conceptos poco conocidos hasta ese entonces en Buenos Aires como los que se desprenden a continuación:

Desde luego, se entiende bien por qué se propagan las especies; porque encerrando el germen en idea el mismo número y género de funciones que el tronco de donde sale, esta idea en su desenvolvimiento debe dar por resultado el mismo género y número de órganos. Pero lo que más interesa y mayores dificultades ofrece, es la explicación de la trasmisión de peculiaridades individuales. En ella vamos a entrar, comenzando por el estado fisiológico, y haciendo después una revista analítica de las enfermedades, que deben ser, y son, en efecto, hereditarias (Rawson, 1845, p.24-25). 
En este párrafo, se observa cómo el problema se ha desplazado respecto de la propuesta de Alcorta: lo importante no es identificar, observar y clasificar las variaciones de las facultades físicas y mentales de los pacientes, sino analizar su transmisión. Según el galeno, el estado de los órganos cerebrales influye también para hacer presentar en la progenie, de un modo permanente, cualidades determinadas primero por una estimulación del momento, o si se quiere, artificial en el encéfalo de los padres. En tal sentido, aparece el término herencia pensado en clave de una comunicación vital. Según Rawson, la herencia asume tres momentos significativos. El primero se aloja al tiempo mismo de empezar la existencia orgánica del "germen"; el segundo, durante la etapa de la gestación, y el tercero, durante la lactancia. En estos tres períodos existe pues una "correlación vital entre los padres y el hijo" (Rawson, 1845, p.27). De tal manera, las facultades intelectuales particulares y los estados morales de los padres se trasladan vía la herencia a los hijos. La herencia actúa en el tránsito de una determinada formación moral de los sujetos porque ésta se constituye a partir de una transmisión cerebral entre padres-hijos. Al mismo tiempo, también las enfermedades fisiológicas se heredan de los antepasados: "No hay una sola de las infinitas dolencias que afligen al hombre, que no se encuentre reproducida hereditariamente" (p.30). Pero no solo la herencia es retrospectiva, o mejor dicho, descendente, sino también es determinante del presente y del futuro, en tanto recala en la ascendencia del progenitor (p.33-34).

Para comprobar pues la influencia del estado moral en las cualidades de la progenie y/o en el "traslado" de las enfermedades, Rawson se apoya en una serie de argumentos de diversa índole. El primero, surge de una historia que le ha llegado a sus oídos por un profesor que conocía. El relato de Rawson comienza refiriendo a un niño que reside en Buenos Aires y que, recién comenzando su enseñanza elemental, se destaca notablemente por la facilidad en que resuelve problemas matemáticos. La explicación del fenómeno versa en la indagación de las circunstancias en las cuales sus padres tuvieron al hijo: en la época de la concepción el padre estaba preocupado por un negocio de importancia que tenía que resolver, calculando diariamente, las ventajas y desventajas de una compra que se le proponía (Rawson, 1845, p.2728). El argumento de las "circunstancias de los padres" extraído de la vida cotidiana porteña posteriormente es asociado a un caso de mayor significación. Rawson (1845, p.28) comenta que "Leticia Ramolini llevaba en su seno al futuro Emperador de la Francia, el conquistador moderno, cuando acompañaba a su esposo Carlos Bonaparte en las gloriosas luchas de su patria". Al retomar este último episodio "histórico", Rawson otorga mayor significación en el consejo de que en la historia de vida de todos los hombres, siempre existe un lazo remarcable concerniente a los padres.

Pero hasta aquí el galeno busca convencer trayendo a colación historias de terceros o basándose en datos históricos conocidos. Nos parece más sugerente tratar de analizar cuáles fueron específicamente los saberes médicos que selecciona para fundamentar su propuesta. Rawson (1845, p.28-29) desarrolla una línea argumental ligada a distintos saberes médicos cuando introduce la siguiente comparación:

El africano y el europeo, tan diferentes por su color como por su inteligencia, tienen un mismo origen. Pero a los primeros sucedió que el clima abrasador en donde habitaban 
les convidada al reposo total de sus facultades, de donde resultó una lenta pero eficaz degeneración de su raza, hasta llegar el estado de miseria en que hoy se nos presentan, casi confundidos con los irracionales, por lo mezquino y material de sus instintos. El europeo, por el contrario, se vio rodeado de necesidades a que era forzoso satisfacer con la industria y el trabajo, y de entonces data esa mejora hoy tan rápida, gracias a los regalos de la civilización ... He aquí, pues, los efectos del estado accidental de ocio en que vive la inteligencia de aquellos pueblos salvajes, pues comunicándose a los descendientes en su mayor entorpecimiento posible, el cerebro va embotándose de generación en generación, como si un peso enorme le aplastara poco a poco.

En las palabras anteriores se sintetizan distintos saberes provenientes de la medicina que circularon en Buenos Aires por ese entonces. ${ }^{5}$ En primer lugar, el registro que corresponde a la primacía de los órganos y su funcionamiento - y el cerebro en particular -, a las facultades intelectuales y a la sensibilidad (derivados de la medicina vitalista y de la filosofía sensualista de la idéologie). Luego, el que reposa en las cuestiones del ambiente, la higiene pública y personal, los climas (proveniente de tradiciones médicas más antiguas como hipocrática y la galénica) y, por último, el que el mismo Rawson promovió sobre la herencia, la predisposición, la degeneración en donde resulta que la clase de vida que posee el germen del nuevo organismo está determinada por la naturaleza vital del cuerpo de que procede. ${ }^{6}$ Se observa como coexisten registros discursivos distintos, pero que situados en esta tesis buscan fundirse en un mismo nivel interpretativo. Y digo "buscan" porque pese al esfuerzo de Rawson en hacerlos compatibles e inteligibles se hace evidente ciertas tensiones entre los mismos.

En especial, en cuanto a aquellas particularidades de los sujetos porque entra en colisión si éstas se derivan únicamente a través de la herencia o más bien se originan por cuestiones ligadas a la higiene y a los influjos del clima. Este problema ciertamente no queda resuelto en la explicación del galeno sanjuanino. La solución momentánea y provisoria que da Rawson, en algunos párrafos, no en todos, es colocar en primer lugar a los condicionamientos ambientales en la conformación de ciertas conductas y en la aparición de determinadas enfermedades; luego, actuaría la herencia como transmisora de esas mismas propiedades iniciales de generación en generación. Surge pues el problema de combinar los conocimientos que provenían de la tradición galénica con los relativos a la predisposición (Lindemann, 2010, p.99-112; González Recio, 2016, p.104-105).

Cabe aclarar que aún en este momento del desarrollo de la medicina resulta difícil distinguir lo hereditario de lo ambiental o, si se quiere, cuáles son los dominios de lo hereditario y de la argumentación higiénico-ambiental. Por otro lado, lo hereditario, aún no se inscribe dentro de los parámetros de una cultura científica positivista como sí ocurrirá a finales del siglo XIX. Es por estas razones que Rawson (1845, p.33-34) a veces matiza su postura: por ejemplo, señala la importancia de la familia en el modo de vida elegido para prevenir las futuras enfermedades de los hijos.

Pero también aquí se hace evidente otra cuestión, por cierto, no menor. Rawson deja atrás las afirmaciones referidas al individuo - sea el niño porteño o el proceso de gestación del futuro Napoleón -, para comenzar a reflexionar sobre lo colectivo. Asimismo, en este registro aparecen enunciados que sí ubican a la herencia como un fenómeno determinante. 
En la cita anterior, se observa cómo introduce vocablos tales como "europeo", "africano", "raza", "pueblos". De tal forma, la herencia no solo conformaría ciertas subjetividades, modos de ser del sujeto y la proliferación de enfermedades propias, sino que también puede pensarse en función de comprender los caracteres de las sociedades, la perfectibilidad de los pueblos, la mejora y retroceso de los grupos humanos. Mientras que el peso de la herencia en el nivel individual podría presentar algún tipo de variación a partir de una terapéutica familiar, en el plano de los pueblos, en cambio, no se otorga esta posibilidad. Como dice más arriba, el pueblo africano está recluido a una "eficaz degeneración de su raza".

Lo moral así comprendido, ya no sólo se piensa como fisiología aplicada vía la imitación de terceros en el marco de las relaciones interpersonales como en el caso de Alcorta, sino que a partir de ligarla a la cuestión de la predisposición se introduce un argumento orgánico-interno (el germen de los padres en el nuevo organismo) capaz de interpretar a las conductas morales como prácticas hereditarias. En su tesis, Rawson despliega el tópico de la herencia configurando una fórmula explicativa bajo la cual lo moral resulta un proceso vital determinado. Lo moral se detiene y se recluye en las posiciones subordinadas de los progenitores, con lo que se produce un escaso margen en los cursos de acción de los hijos, postura que más adelante será ampliamente difundida y sostenida en el relato galénico de fines del siglo XIX. Asimismo, esta moral en clave de herencia, al tener la posibilidad de precisar conexiones entre los temperamentos y observar continuidades en los malestares, puede servir para construir perfiles de las conductas sociales y construir tablas de enfermedades frecuentes.

\section{Francisco Javier Muñiz, entre el clima y los vientos}

Por su parte, también Francisco Javier Muñiz cuando escribía sobre medicina e historia natural añadía ciertas formulaciones y temáticas de índole moral y social, aunque en este caso, ya no son los órganos ni la herencia los que propician determinadas formas de actuar en el hombre. En el relato de Muñiz más bien aparece una nueva pero antigua explicación que estriba en introducir el tema de la gravitación que ejerce la naturaleza y, en especial los vientos, en la vida diaria de las personas.

Muñiz nació en Monte Grande, en la provincia de Buenos Aires, el 21 de diciembre de 1795. Estudió en el Instituto Médico Militar, fundado por el doctor Cosme Argerich para formar cirujanos que sirvieran al ejército. Se graduó de médico en 1822, cuando el Instituto ya formaba parte de la Universidad de Buenos Aires, creada el año anterior. Desde enero de 1825 fue cirujano de la guardia de Chascomús. Allí, se dedicó a la observación de la naturaleza, investigando la flora y fauna locales. Cuando en 1826 estalló la Guerra del Brasil, fue nombrado médico y cirujano principal del ejército, con el grado de teniente coronel.

En 1828 se instaló en la ciudad de Luján y fue nombrado como administrador de la vacuna antivariólica. Allí, continúo sus investigaciones paleontológicas en su tiempo libre, extrayendo de las barrancas del río una extraordinaria serie de fósiles. Por sus propios medios, Muñiz exhumó restos de varias especies animales extinguidas, unas ya conocidas y otras descubiertas por primera vez; las reconstruyó y estudió con cuidado. Entre sus hallazgos figuran mastodontes, megaterios, gliptodontes, caballos y tigres fósiles 
(Podgorny, 2007, 2010). En base a estos descubrimientos fue que estableció un vínculo epistolar importante con Charles Darwin (Novoa, Levine, 2010, p.36-37).

En 1847, Muñiz terminó su obra Apuntes topográficos del centro de la provincia de Buenos Aires. El texto es una reseña topográfica que analiza la composición del suelo y de la geología, la incidencia del clima, la alimentación y el trabajo. Pero también incluye el estudio de las características físicas y morales de los habitantes junto a las enfermedades más frecuentes. Al respecto, Muñiz (1944, p.236) indica que: "Conocemos cuán difusa es y fecunda en observaciones una información sobre la atmósfera y los varios agentes que obran en ella".

Evidentemente, la propuesta tenía antecedentes. En la Antigüedad, Aristóteles se había ocupado de la influencia moral de los climas (Aristóteles, 1988, l.VII, c.III, IV, V, VI). También este fundamento antiguo se había reintroducido recientemente por algunos pensadores ilustrados como Montesquieu quien en 1748 con la publicación del El espíritu de las leyes explicó la estrecha relación que existía entre los climas, la geografía y los tipos de estados (Montesquieu, 1995, 1, XIV, XV, XVI, XVII).

En el caso de Muñiz, no obstante, aparece un componente novedoso. Me refiero a la presencia de una nueva intermediación que buscó construir una argumentación más sólida en torno a la relación espacio/hombre. Esta interposición, que agregaría un tercer elemento aglutinador en los fundamentos, proviene de la medicina de la época, en especial, como ya apreciamos en los análisis sobre Alcorta y Rawson, de la escuela sensualista, del vitalismo y la temprana fisiología a partir de la incorporación de aquellas nociones difundidas sobre el funcionamiento de los órganos, la fuerza vital y la formación de las ideas a partir de la sensibilidad.

En esta visión, los climas alcanzan el funcionamiento de los órganos y con ello también a las acciones morales de los hombres. Al determinismo climatológico de Montesquieu, Muñiz agrega un razonamiento de tipo orgánico y del sistema nervioso, un determinismo pues que como dispositivo de argumentación buscó articular lo externo y lo interno, el entorno y el cuerpo, lo inorgánico y lo orgánico. Muñiz (1944, p.227) explica que "en cuanto a las cuatro condiciones primeras de los vientos, su humedad o sequedad, su frigidez o calorificación ejercen aquí, como en todas partes, una influencia directa sobre los cuerpos".

Según Muñiz, el clima y la acción de los vientos trastocan el sistema nervioso de los sujetos afectando la sensibilidad de los cuerpos y el estado de los órganos. De tal modo, aparecen determinados síntomas y luego una serie de enfermedades que el médico (también el político) podría registrar detalladamente como se sigue a continuación:

El Norte frío y húmedo en el invierno, entorpeciendo la potencia nerviosa, disminuyendo su actividad, causando una sedación en sus propiedades, excita o predispone a los efectos nerviosos ya enunciados. Al mismo tiempo que relaja y comprime la toracidad de las fibras, debilita la epidermis y la energía de la vida exterior, haciendo muy sensibles los cuerpos. Entonces acaecen los efectos reumatismales, los dolores sobre varias partes etc. Se observa que el Norte en este estado irrita el sistema nervioso de aquellas personas en quienes predomina sobre los demás. Se ve en la Capital, pues en la campaña son casi desconocidas estas afecciones, que los accesos histéricos, los hipocondríacos, la manía, ciertas neuralgias, son como provocadas por este viento (Muñiz, 1944, p.228-229). 
Este efecto del clima en los órganos, permitiría establecer y verificar comportamientos uniformes y estables en una determinada población. Y una vez más, pasamos de lo individual a lo colectivo, pero esta vez no se resalta la explicación de la imitación ni la gravitación de la herencia sino la acción de los vientos y su injerencia en el sistema nervioso. De esta interacción entre lo orgánico y lo natural surge el tópico moral. En tal sentido, el estudio de las propiedades naturales de los vientos y su ubicación espacial servirían para identificar a las poblaciones con mayor propensión a conductas asociadas a la transgresión y a la criminalidad. Tal como indica Muñiz en las siguientes palabras:

El Norte húmedo y caliente excita la irascibilidad en los individuos de temperamento nervioso o hepático. La experiencia ha demostrado que los crímenes más atroces, aquellos que se comenten por la exaltación de una pasión del momento, por la furiosa explosión de un sentimiento cruel y sanguinario, tienen lugar en su mayor número, reinando el Norte, mucho más si ha sido por varios días consecutivos (Muñiz, 1944, p. 229).

El párrafo anterior es representativo de cómo los saberes médicos se piensan en cuanto a que éstos pueden servir como insumo para la reflexión de las representaciones morales y sociales. La concentración de las pasiones y la sensibilidad, acentuada por los climas, genera sujetos crueles y sanguinarios cuyas conductas se conforman a partir de la rebeldía y la violencia. El norte provoca la irascibilidad moldeando sujetos que no ajustan sus comportamientos a la ley, la obediencia, la jerarquía.

La tesis en torno a que los vientos activan el juego de los órganos, concentran la sensibilidad, exaltan el sistema nervioso y perturban las funciones del sensorio, genera una interpretación en donde las acciones físicas y morales quedan equiparadas. El estudio de los vientos y los diferentes climas se concibe como un programa central a la hora de conocer y detallar las características de una determinada población. Para Muñiz, la historia del medio ambiente, es decir, de cuanto nos rodea o influye sobre nuestros órganos, es fundamental para conocer las primeras cualidades de sus habitantes, la propensión de éstos a determinadas enfermedades, y al esquema moral de un grupo social.

En suma, a la medicina le toca hacer conocer aquellos elementos que alteran particularmente el estado de la sensibilidad del hombre, y determinar cuáles son los medios cuya acción pueda restituirla al orden natural. Puesto bajo este punto de vista el estudio de los vientos y los climas resulta primordial tanto para el filósofo como para el gobernante. Para Muñiz, aquí es donde pueden encontrar nuevos conocimientos sobre la naturaleza humana y hacer observaciones fundamentales sobre su "perfección".

\section{Consideraciones finales}

En primer lugar, se buscó reconstruir algunos rasgos generales del orden rosista e identificar un conjunto de reflexiones sobre la vida académica y médica en este período. En esta etapa se profundizó un proceso de politización de la vida social y política por las características propias del poder concentrado que ejerció Rosas y por las guerras externas y civiles desatadas, alcanzando al mundo de la medicina. A pesar de las modificaciones 
institucionales, los conflictos dentro del mismo cuerpo médico, las reglamentaciones que obligaron seguir la "causa federal" en la Universidad, los problemas del contexto económico por las guerras y, sobre todo, las tensiones y conflictos abiertos por el faccionalismo partidario, los médicos tuvieron un papel relativamente activo en ese entonces.

Algunos galenos experimentaron las consideraciones del gobierno; otros, en cambio, tuvieron que renunciar a sus cargos profesionales e incluso se vieron obligados a emigrar. En este marco, encontramos que Diego Alcorta, Guillermo Rawson y Francisco Muñiz, atravesados por la vida política de aquel entonces, optaron por realizar distintas reflexiones de índole moral a partir de los conocimientos médicos en los cuales se habían formado y que procedían de las tradiciones médicas europeas, en especial, la francesa.

Se ha analizado cómo surgen afirmaciones y razonamientos que convergen en generar líneas argumentales comunes en torno a colocar al hombre en tanto un ser vivo, orgánico, vital, sensible. Evidentemente, la escuela vitalista y la fisiología francesa tuvieron una presencia importante en la medicina porteña de las primeras décadas del siglo XIX. Pero, también es cierto que al mirar con mayor detenimiento los escritos aparecieron variaciones en cuanto a cómo los médicos locales construyeron con las mismas premisas compartidas distintas formas de comprender lo moral.

Alcorta retomó la cuestión de los órganos y la sensibilidad para introducir y resaltar el componente del ejemplo social. El funcionamiento interno se relaciona con lo externo mediante el ejemplo exterior, son las conductas sociales y las formas de ser de otros hombres los que conducen a comprender la moral: de allí la introducción de la teoría de la imitación. En cambio, para Rawson, la dinámica de los órganos se conectó más bien con el problema de la herencia con lo cual lo moral se piensa como algo propiamente interno y determinado. Lo moral se "traslada" de padre a hijo porque las conductas que se originan guardan relación con las predisposiciones de la progenie. Una tercera perspectiva se sitúa a través del análisis del escrito de Muñiz, el cual ligó el postulado de los órganos y de la sensibilidad a las situaciones externas de la naturaleza, la atmosfera, los climas y los vientos que son los que repercuten en el tipo de moral de un individuo y población.

Ahora bien, en estos textos, las prácticas morales comienzan a pensarse en torno a la materia misma y no ya como parte de una fuerza espiritual y superior a ella, pero paradójicamente, debido al avance de estos conocimientos médicos, se desarrolla la idea de que el hombre no puede controlar del todo el curso de sus conductas ya que éstas no dependen exclusivamente de su voluntad y deseos. Aparecen condicionantes que deben tenerse en cuenta: el contacto con otros sujetos al que la posibilidad de imitación queda restringida al contexto donde uno ha nacido o se ha desarrollado (Alcorta); la fuerza oculta del germen de la herencia que aparece en el nuevo organismo como inalterable (Rawson) y/o por la gravitación de la naturaleza y los efectos del ambiente (Muñiz). Estos factores producen en conjunto una representación de lo moral en términos de un itinerario humano cada vez menos dependiente de sí mismo.

Por otro lado, el análisis de estos escritos también nos indicó el surgimiento de explicaciones morales como un fenómeno colectivo, es decir, una moral que puede ser socialmente estudiada y tipificada. Este asunto nos sugiere continuar indagando, porque si lo moral es plausible de aplicarse a lo colectivo, los saberes galénicos aparecerían como 
contenidos útiles y prácticos, puestos como recursos o insumos no solo del propio avance médico sino también respecto de un posible "uso" político. Estos saberes médicos podrían resultar funcionales en cuanto a la regulación de las pasiones en la vida política como sucedió en Inglaterra y Francia en los siglos XVIII y XIX (Moscoso, 2010).

El fenómeno por el cual algunos médicos representativos de la etapa rosista comenzaron a escribir y a fijar su atención en cuestiones de índole moral en los grupos humanos nos propone introducir en futuras investigaciones el problema de cómo este fenómeno podría tener cierta articulación con lo que algunos autores han denominado la emergencia de la "temprana cuestión social" (González Leandri, González Bernaldo, Suriano, 2010).

\section{NOTAS}

${ }^{1}$ El vitalismo se caracterizaba por postular la existencia de una fuerza o un impulso vital sin el cual la vida no podría ser argumentada. Tras retomar las ideas del médico y químico alemán Georg Ernst Stahl (1660-1734), sus miembros creían que el alma era el principio vital que controlaba el desarrollo orgánico. El principal difusor del vitalismo en Francia fue Xavier Bichat. Él entendió que los fenómenos biológicos tenían por causa propiedades vitales innatas a todos los seres vivos. De allí derivó el límite que impuso en sus estudios: observó los hechos sin profundizar más sus investigaciones, pues estaba convencido de que ni la física ni la química podían interpretar la vida. Como se desprende de su obra Recherches physiologiques sur la vie et la mort, publicada en 1800, Bichat (1862) defendió la irreductibilidad de la vida a la materia inerte. Esta postura lo llevó a desconfiar del uso del microscopio y considerar la disección como forma de demostración e impulsar los estudios histológicos. Tras su muerte, en 1802, su discípulo François Magendie imprimió otra dirección en las ideas de su maestro. Para Magendie los fenómenos orgánicos son susceptibles de ser reducidos a explicaciones físicas y químicas y todas sus demostraciones experimentales tendieron a probar este aserto. Esta diferenciación permitió el desarrollo y la construcción de otra rama que empezó a tener suma importancia en la época: la fisiología experimental (Le Blanc, 2004, p.1210; Normandin, 2007, p.495-528).

${ }^{2}$ En el caso de las Investigaciones fisiológicas de la vida y la muerte de Bichat se trata seguramente de la traducción al castellano hecha por el médico Tomás García Suelto, publicada por la Imprenta de la Administración del Real Arbitrio, en Madrid en 1806. En el caso de Pinel, el testamento indica que se trataría de la primera edición en francés: La médecine clinique rendue plus précise et plus exacte par l'application de l'analyse, ou Recueil et résultat d'observations sur les maladies aiguës à la Salpêtrière. Paris, 1802.

${ }^{3}$ Pierre Jean Georges Cabanis nació el 5 de junio de 1757 en Cosnac (Francia) y murió el 5 de mayo de 1808. Estudió medicina en París. A través del contacto que mantenía con la viuda de Helvetius conoció a Diderot, d'Holbach y Condorcet. Su método consistió en aislar los elementos, para caracterizarlos mejor y deducir diagnósticos sobre lo físico y moral humano. En 1802, publicó Rapparts du physique et du moral de l'homme, donde explica las relaciones de la moral con las edades, sexos, razas, climas. Realizó estudios de las sensaciones externas e internas, del sistema nervioso, de la fisiología cerebral, del pensamiento, de las pasiones (Staum, 1980; Besançon, 1997).

${ }^{4}$ Philippe Pinel nació el 20 de abril de 1745 en Saint-Paul-Cap-de-Joux (Francia) y murió el 25 de octubre de 1826. Estudió en Toulouse y Montpellier. Se dedicó al estudio y tratamiento de las enfermedades mentales. Fue uno de los que consideró la clínica médica como observación y análisis sistemático de los fenómenos perceptibles de la enfermedad. Propició la humanización del trato que se daba por entonces a las personas aquejadas por las enfermedades mentales, eliminando, su encerramiento. Consideró posible la recuperación de un amplio grupo de los alienados a partir de un tratamiento moral (Lesch, 1984; Weiner, 1999).

${ }^{5}$ En trabajos anteriores, demostramos con mayor profundidad y espacio el ingreso de distintas corrientes médicas francesas en Buenos Aires hacia 1820 (Di Pasquale 2014b, 2015).

${ }^{6}$ Los primeros debates sobre la herencia se dieron en Francia a partir de 1790, en la Société Royale de Médecine de París, que lanzó un concurso donde se debía responder si efectivamente existían enfermedades hereditarias. Las respuestas quedaron divididas en dos posiciones: aquellos que pensaban a las patologías hereditarias a partir de causas o problemas en los humores, en especial, a través de virus o gérmenes y aquellas tesis que postulaban e incorporaban la noción de predisposición. Este debate será ampliado y atravesará todo el desarrollo de la medicina durante el siglo XIX, hasta llegar a Francis Galton que impondrá el predominio de la segunda opción (Vallejo, 2012). 


\section{REFERENCIAS}

ALCORTA, Diego.

Lecciones de filosofía. Buenos Aires: Fondo

Nacional de las Artes. [1835] 2001.

ALCORTA, Diego.

Diario literario por la Sociedad Elemental de Medicina. Colección Candioti. Sala del Tesoro, ref.3A63519. (Biblioteca Nacional Mariano Moreno, Argentina). 1821.

ALCORTA, Diego.

Carta a Daniel Torres. In: Sala VII.

Documentación de Daniel Torres. leg. 1943

(Archivo General de la Nación, Argentina). 27 dic. 1834.

ALCORTA, Diego.

Disertación sobre la manía aguda. Colección Candiotti. Sala del Tesoro, ref.3A232210.

(Biblioteca Nacional Mariano Moreno,

Argentina). 1827.

ARGERICH, Cosme Mariano.

Testamento. In: Sala IX. Sucesiones, leg. 3475

(Archivo General de la Nación, Argentina). 1820.

ARISTÓTELES.

La Política. Madrid: Editorial Gredos. 1988.

ARMUS, Diego.

La ciudad impura: salud, tuberculosis y cultura en Buenos Aires, 1870-1950. Buenos Aires: Edhasa. 2007.

BESANÇON, Serge.

La philosophie de Cabanis: une réforme de la psychiatrie. Paris: Institut Synthélabo pour le progrès de la connaissance. 1997.

BICHAT, Xavier.

Recherches physiologiques sur la vie et la mort.

Paris: Librairie Victor Mason et Fils. 1862.

BONNER, Thomas.

Becoming a physician: medical education in

Britain, France, Germany, and the United States, 1750-1945. Oxford: Oxford University Press. 1995.

BUCHBINDER, Pablo.

Historia de las universidades argentinas. Buenos Aires: Sudamericana. 2005.

BYNUM, William.

Science and the practice of medicine in the nineteenth century. Cambridge: Cambridge University Press. 2006.

CABANIS, Pierre Jean Georges.

Rapports du physique et du moral de l'homme. Paris: L'Harmattan, 2v. 2005.

CONDILLAC, Étienne Bonnot de.

Langue des calculs. Paris: Librarie Sandoz et Fischbacher. 1877.
CUENCA, Claudio Mamerto.

Discurso. In: Rawson, Guillermo. Disertación para obtener el grado de doctor en medicina para la Universidad de Buenos Aires. Buenos Aires: Imprenta de la Independencia. p.12-18. 1845.

CUTOLO, Vicente Osvaldo.

Nuevo diccionario biográfico argentino: 1750-1930. Buenos Aires: Elche, v.4. 1958-1985.

DI LISCIA, María Silvia.

Saberes, terapias y prácticas médicas en Argentina (1750-1910). Madrid: CSIC. 2002.

DI LISCIA, María Silvia; SALTO, Nélida Graciela (Eds.).

Higienismo, educación y discurso en la Argentina (1870-1940). Santa Rosa: Universidad Nacional de La Pampa. 2004.

DI PASQUALE, Mariano.

Vitalismo, idéologie y fisiología en Buenos Aires. La polémica entre Cosme Mariano Argerich y Crisóstomo Lafinur en El Americano, 1819. Revista Ciencias de la Salud, v.13 (especial), p.13-28. 2015.

DI PASQUALE, Mariano.

Diego Alcorta y la difusión de saberes médicos en Buenos Aires, 1821-1842. Dynamis, Acta Hispanica ad Medicinae Scientiarumque Historiam Illustrandam, v.34, n.1, p.125-146. 2014a.

DI PASQUALE, Mariano.

La présence de l'idéologie à Buenos Aires. Un élan philosophique dans le processus de professionnalisation de la médecine, 1820-1840. Cahiers des Amériques Latines, n.76, p.111-129. 2014b.

DI PASQUALE, Mariano.

La recepción de la idéologie en la Universidad de Buenos Aires. El caso de Juan Manuel Fernández de Agüero (1821-1827). Prismas, Revista de Historia Intelectual, n.15, p.63-86. 2011.

FERNÁNDEZ DE AGÜERO, Juan Manuel. Principios de ideología. Primer curso de filosofía dictado en la Universidad de Buenos Aires (18221827), 3 t. Buenos Aires: Universidad de Buenos Aires, Facultad de Filosofía y Letras. 1940.

GAUDILLIÈRE, Jean-Paul.

La médecine et les sciences, XIX-XX siècles. Paris: La Découverte. 2006.

GOBIERNO...

Gobierno de la Provincia de Buenos Aires. Reglamento para la colación de grados en la Universidad, de 21 de junio de 1827. In: De Angelis, Pedro. Recopilación de leyes y decretos promulgados en Buenos Aires desde el 25 de mayo de 1810 hasta el fin de Diciembre de 1835. Buenos Aires: Imprenta del Estado, v.2, p.863-864. 1836a. 
GOBIERNO...

Gobierno de la Provincia de Buenos Aires. Decreto. Suprimiendo el Consejo Directivo de la Universidad, de 11 de mayo de 1835. In: De Angelis, Pedro. Recopilación de leyes y decretos promulgados en Buenos Aires desde el 25 de mayo de 1810 hasta el fin de Diciembre de 1835. Buenos Aires: Imprenta del Estado, v.2, p.1273. 1836b.

\section{GOBIERNO...}

Gobierno de la Provincia de Buenos Aires. Decreto. Fórmula de todo juramento que se debe prestar públicamente, de 20 de junio de 1835. In: De Angelis, Pedro. Recopilación de leyes y decretos promulgados en Buenos Aires desde el 25 de mayo de 1810 hasta el fin de Diciembre de 1835. Buenos Aires: Imprenta del Estado, v.2, p.1283. 1836c.

\section{GOBIERNO...}

Gobierno de la Provincia de Buenos Aires. Decreto. Reorganizando la Universidad, de 14 de diciembre de 1835. In: De Angelis, Pedro. Recopilación de leyes y decretos promulgados en Buenos Aires desde el 25 de mayo de 1810 hasta el fin de Diciembre de 1835. Buenos Aires: Imprenta del Estado, v.2, p.1330-1331. 1836d.

GOBIERNO...

Gobierno de la Provincia de Buenos Aires. Decreto. Requisitos que se prescriben para recibir el grado de Doctor, de 27 de enero de 1836. Registro Oficial de la Provincia de Buenos Aires. Buenos Aires: Imprenta del Estado, 1.15, p.14. 1836e.

GONZÁLEZ BERNALDO DE QUIRÓS, Pilar. Civilidad y política en los orígenes de la nación argentina: las sociabilidades en Buenos Aires, 1829-1862. Buenos Aires: Fondo de Cultura Económica. 2008.

GONZÁLEZ LEANDRI, Ricardo.

Curar, persuadir, gobernar: la construcción histórica de la profesión médica en Buenos Aires (1852-1886). Madrid: CSIC. 1999.

GONZÁLEZ LEANDRI, Ricardo; GONZÁLEZ BERNALDO, Pilar; SURIANO, Juan.

La temprana cuestión social: la ciudad de Buenos Aires durante la segunda mitad del siglo XIX. Madrid: CSIC. 2010.

GONZALEZ RECIO, José Luis.

Los hechos y las hipótesis en la fisiología francesa del siglo XIX. Ludus Vitalis, v.24, n.45, p.101-126. 2016.

GRMEK, Mirko (Dir.).

Histoire de la pensée médicale en Occident. Paris: Seuil, v.3.1997.

GUTIÉRREZ, Juan María.

Noticias históricas sobre el origen y desarrollo de la enseñanza pública superior en Buenos Aires, 1868. Bernal: Universidad Nacional de Quilmes. 1998.
HABER, Samuel.

The quest for authority and honor in the American professions, 1750-1900. Chicago: Chicago

University Press. 1991.

HALPERIN DONGHI, Tulio.

Historia de la Universidad de Buenos Aires. Buenos Aires: Eudeba. 1962.

ISABELLE, Arsène.

Viaje a la Argentina, Uruguay y Brasil, 1830-1834. Buenos Aires: Emecé. 2001.

LA ABEJA...

La Abeja Argentina (1822-1823). In: Biblioteca de Mayo. Colección de Obras y Documentos para la Historia Argentina, t.6: literatura. Buenos Aires: Senado de la Nación. p.5245-5700. 1960.

LAFINUR, Juan Crisóstomo.

Curso filosófico. Buenos Aires: Facultad de Filosofía y Letras de la Universidad de Buenos Aires. 1939.

LE BLANC, Guillaume.

Vitalisme (École de Montpellier). In: Lecourt, Dominique (Ed.). Dictionnaire de la pensée médicale. Paris: Press Universitaires de France, p.1208-1211. 2004.

LESCH, John.

Science and medicine in France: the emergence of experimental physiology, 1790-1855.

Cambridge: Harvard University Press. 1984.

LINDEMANN, Mary.

Medicine and society in early modern Europe.

Cambridge: Cambridge University Press. 2010.

MONTESQUIEU.

El espíritu de las leyes. t.1. Paris: Folio Essais. 1995.

MOSCOSO, Javier.

Materialismo y religión: ciencias de la vida en la Europa ilustrada. Barcelona: Ediciones del Serbal. 2010.

MUÑIZ, Francisco Javier.

Apuntes topográficos del territorio y adyacencias del departamento del centro de la provincia de Buenos Aires, con algunas referencias a lo demás de su campaña. In: Muñiz, Francisco Javier. Escritos científicos. Buenos Aires: W.M. Jackson, p.198-243. 1944.

MYERS, Jorge.

Orden y virtud: el discurso republicano en el régimen rosista. Bernal: Universidad Nacional de Quilmes. 1995.

NORMANDIN, Sebastian.

Claude Bernard and an introduction to the study of experimental medicine: "physical vitalism", dialectic, and epistemology. Journal of the History of Medicine and Allied Sciences, n.62, p.495-528. 2007. 
NOVOA, Adriana; LEVINE, Alex.

From man to ape: Darwinism in Argentina, 18701920. Chicago: Chicago University Press. 2010.

PARADA, Alejandro.

El mundo del libro y de la lectura durante la época de Rivadavia: una aproximación a través de los avisos de La Gaceta Mercantil (1823-1828). Cuadernos de Bibliotecología, n.17, p.1-174. 1998.

PESTRE, Dominique.

Pour une histoire sociale et culturelle des sciences, nouvelles définitions, nouveaux objets, nouvelles pratiques. Annales HSS, n.3, p.487-522. 2005.

PICKERING, Andrew (Ed.).

Science as practice and culture. Chicago: Chicago University Press. 1992.

PINEL, Philippe.

Traité médico-philosophique sur l'aliénation mentale, ou la manie. Paris: Richard, Caille et Ravier. 1801.

PODGORNY, Irina.

Los médicos de muertos y la paleontología en la Plata: medicina legal, cirugía militar y observación de campo en la obra de Francisco X. Muñiz, 1830-50. Anuario IEHS, n.25, p.303-352. 2010.

PODGORNY, Irina.

De ángeles, gigantes y megaterios. Saber, dinero y honor en el intercambio de fósiles en las provincias del Plata en la primera mitad del siglo XIX. In: Salvatore, Ricardo (Ed.). Los lugares del saber: contextos locales y redes transnacionales en la formación del conocimiento moderno. Rosario: Beatriz Viterbo. p.125-157. 2007.

RAMSEY, Matthew.

Professional and popular medicine in France 17701830: the social world of medical practice. Cambridge: Cambridge University Press. 1988.

RAWSON, Guillermo.

Bellas artes y ciencia. In: Rawson, Guillermo. Escritos científicos. Buenos Aires: W.M. Jackson, p.1-20. 1927.

RAWSON, Guillermo.

Disertación para obtener el grado de doctor en medicina para la Universidad de Buenos Aires.
Buenos Aires: Imprenta de la Independencia. (Biblioteca Central "Juan José Montes de Oca". Facultad de Medicina, Universidad de Buenos Aires). 1845.

STAUM, Martin.

Cabanis: Enlightenment and medical

philosophy in the French revolution. Princeton: Princeton University Press. 1980.

TERNAVASIO, Marcela.

La revolución del voto. Política y elecciones en Buenos Aires, 1810-1852. Buenos Aires: Siglo XXI 2002.

TERNAVASIO, Marcela.

Hacia un régimen de unanimidad: política y elecciones en Buenos Aires, 1828-1850. In: Sabato, Hilda (Ed.). Ciudadanía política y formación de las naciones. México: Fondo de Cultura Económica, p.119-141. 1999.

VALLEJO, Mauro.

Teorías hereditarias del siglo XIX y el problema de la transmisión intergeneracional. Tesis (Doctorado en Psicología) - Universidad de Buenos Aires, Buenos Aires. 2012.

VAN DAMME, Stéphane.

Histoire des sciences et des techniques. In:

Delacroix, Christian et al. Historiographies, t.1. Paris: Gallimard. p.242-254. 2010.

VIGARELLO, Georges.

Histoire des pratiques de santé. Paris: Éditions du Seuil. 1993.

\section{WEINER, Dora.}

Comprende et soigner: Philippe Pinel (1745-1826)

- la médicine de l'esprit. Paris: Fayard. 1999.

YRIGOYEN, Manuel.

Comunicación del Ministerio de Gobierno al Ministerio de Hacienda. Tribunal de Medicina. 1.10-6-6-2A (Archivo General de la Nación, Argentina). 24 abr. 1835.

ZIMMERMANN, Eduardo.

Racial ideas and social reform: Argentina, 18901916. The Hispanic American Historical Review, v.72, n.1, p.23-46. 1992.

\section{$\rightarrow \rightarrow \rightarrow<<$}

